\title{
Risk of dementia in people with depressive and bipolar disorders increases with increasing number of prior affective episodes
}

Kessing LV, Andersen PK. Does the risk of developing dementia increase with the number of episodes in patients with depressive disorder and in patients with bipolar disorder? J Neurol Neurosurg Psychiatry 2004;75:1662-6.

Does an increasing number of prior affective episodes increase the risk of developing dementia in people with depressive and bipolar disorders?

\section{METHODS}

Design: Cohort study.

Follow up period: 14 years.

Setting: Denmark from 1970 to 1999

of People: 18726 people with depressive disorder (ICD-8) and 4248 people with bipolar disorder (ICD-10). Inclusions: people who at their first discharge from a psychiatric hospital after 1 January 1970 had a diagnosis of primary affective disorder. Main exclusions: diagnosis of organic psychosis or schizophrenia at discharge before 1 January 1986

$\Delta$ Risk factors: The Denmark nationwide register was used to identify participants' psychiatric ward admissions and discharge diagnoses. The relation between number of prior hospital admissions and subsequent dementia diagnosis was estimated using a Cox proportional hazards regression model.

\section{Five}

\section{MAIN RESULTS}

There was an overall trend towards increasing risk of dementia with increasing number of prior episodes of hospitalisation for depressive disorder or bipolar disorder $(\mathrm{p}<0.001)$. This effect was greater in people with depressive disorder than in people with bipolar disorder $(\mathrm{p}<0.05)$. In people with depressive disorder, five or more episodes significantly increased risk of dementia compared with a single episode (hazard ratio 6.16, 95\% CI 1.39 to 27.22 ). On average, each additional episode increased the rate of dementia diagnosis by $13 \%$

For correspondence: L V Kessing, Department of Psychiatry, University Hospital of Copenhagen, Rigshospitalet, Blegdamsvej 9, DK 2100 Copenhagen $\varnothing$, Denmark; lars.kessing@rh.dk

Sources of funding: Stanley Medical Research Institute and Lundbeck Foundation.

(95\% CI $9 \%$ to $16 \%$ ) in people with depressive disorder and by $6 \%$ (95\% CI 3\% to $10 \%$ ) in people with bipolar disorder.

\section{CONCLUSIONS}

In people with depressive or bipolar disorder the risk of diagnosis of dementia on subsequent hospitalisation increases with the number of prior hospitalisations for affective episodes.

\section{Commentary}

he future of optimal dementia care may involve the early detection of risk factors for the onset of dementia, facilitating preventive measures aimed at delaying the onset of dementia. Depression has been identified as a probable risk factor for future onset of Alzheimer's disease (AD). While there are methodological constraints on the data, Kessing and Anderson's paper adds to the existing literature by showing that recurrence of depressive episodes may increase the risk of developing dementia. It also adds data in support of the putative increased risk for dementia associated with recurrence of bipolar affective disorder (BAD).

From a clinical point of view, the data suggest that our index of concern for future onset of dementia should increase with recurrences of depression or BAD (at least if associated with hospitalisation). Increased monitoring of people with recurrent depression or BAD, and educating them about the risk of developing dementia (to allow for planning, for example) should be considered. Although we do not yet have conclusive data that available treatments delay the onset of dementia in people with recurrent depression or $B A D$, there are suggestive data about a number of potential therapeutic options. Those at risk of the onset of dementia should be informed of these potential options (along with the limitations of the evidence and the risks associated with their use).

Future research on the pathophysiology of dementia should explore the possibility that depression causes changes in the brain that ultimately lead to dementia. Alternatively, it may be that preclinical changes in the brain associated with $A D$ are causing the depressive episodes. Kessing and Anderson also suggest that future research should explore lowering the risk of dementia through treatment of recurrent depression and BAD.

Robert van Reekum, MD, FRCPC Department of Psychiatry and the Kunin-Lunenfeld Applied Research Unit, Baycrest Centre for Geriatric Care, Department of Psychiatry, University of Toronto, Canada

1 van Reekum R, Simard M, Cohen T. Towards a research agenda: a review of the prediction and prevention of Alzheimer's disease. J Psychiatry Neurosci 1999;24:413-30. 